\title{
Application of AXUV diode detectors at ASDEX Upgrade
}

\author{
M. Bernert, a) T. Eich, A. Burckhart, J. C. Fuchs, L. Giannone, A. Kallenbach, R. M. McDermott, B. Sieglin, and \\ the ASDEX Upgrade team \\ Max Planck Institute for Plasma Physics, EURATOM Association, Boltzmannstr. 2, 85748 Garching, \\ Germany
}

In the ASDEX Upgrade tokamak a radiation measurement for a wide spectral range, based on semiconductor detectors, with 256 lines of sight (LOS) and a time resolution of $5 \mu$ s was recently installed. In combination with the foil based bolometry, it is now possible to estimate the absolutely calibrated radiated power of the plasma on fast timescales. This work introduces this diagnostic based on AXUV (Absolute eXtended UltraViolet) n-on-p diodes made by International Radiation Detectors, Inc. (IRD). The measurement and the degradation of the diodes in a tokamak environment is shown. Even though the AXUV diodes are developed to have a constant sensitivity for all photon energies ( $1 \mathrm{eV}$ to $8 \mathrm{keV})$, degradation leads to a photon energy dependence of the sensitivity. The foil bolometry, which is restricted to a time resolution of less than $1 \mathrm{kHz}$, offers a basis for a time dependent calibration of the diodes. The measurements of the quasi-calibrated diodes are compared with the foil bolometry and found to be accurate on the $\mathrm{kHz}$ time scale. Therefore, it is assumed, that the corrected values are also valid for the highest time resolution $(200 \mathrm{kHz})$. With this improved diagnostic setup, the radiation induced by edge localized modes (ELMs) is analyzed on fast timescales.

\section{INTRODUCTION}

It is crucial to measure the radiated power in fusion devices, since it represents a significant fraction of the exhaust power and is necessary for power balance calculations. Typically, most of the power is radiated in the divertor region (see Fig. 2), where the plasma is in direct contact with the wall. In this region the electron temperature is relatively low $(10-100 \mathrm{eV})$ and neutral atoms and partially ionized ions can efficiently radiate a significant fraction of the power to the divertor walls.

In the divertor, the radiation is mainly emitted in the vacuum ultraviolet (VUV) region, with photon energies between $5-100 \mathrm{eV}$. Also in the scrape off layer (SOL), the plasma outside of the last closed flux surface (LCFS, see Fig. 2), the radiation is emitted mainly in the VUV region, as a result of the low electron temperature in that region. Inside the LCFS the electron temperatures are higher than $100 \mathrm{eV}$, moving the dominant photon emission to the soft X-ray region $(\sim 100-3000 \mathrm{eV})$.

In most fusion devices, bolometers are used to measure plasma radiation. Bolometers measure the power deposition of electromagnetic radiation and are sensitive to a wide wavelength range from visible light to soft Xray. In this way they measure the total radiated power illuminating the material, but do not retain any spectral information.

Typical bolometers for fusion devices are thin metallic foils (gold or platinum), which are heated by the radiation $^{1-3}$. The time resolution of this kind of detectors is typically in the range of a few milliseconds. Nevertheless, the timescales of many events in a tokamak plasma are significantly shorter.

To achieve a time resolution of a few microseconds, semiconductor based wide spectral range radiation mea-

a) Electronic mail: matthias.bernert@ipp.mpg.de surements are used in other tokamak experiments such as Alcator C-Mod ${ }^{4,5}$, DIII-D ${ }^{6}$ and $\mathrm{TCV}^{7}$. An extensive set of these AXUV diodes was recently installed at the ASDEX Upgrade tokamak (AUG) ${ }^{8}$. Unlike the foil bolometers, the wavelength dependence is not linear for the diodes and the sensitivity degrades in the environment of a tokamak. The sensitivity to the important VUV region is affected particularly severely.

For tokamaks, toroidal symmetry is a generally accepted approximation. Therefore, the total radiation can be measured by installing the bolometer cameras just within one sector (toroidal section) of the torus. However, the new AXUV system includes also one camera which is installed in the toroidally opposing sector for asymmetry studies. With this setup, the toroidal differences of the radiation can be studied (for example, for edge localized modes (ELMs), which are quasi-cyclic instabilities that appear at the edge of the confined plasma in high confinement mode (H-mode) operation ${ }^{9}$.)

The paper is organized as follows. Section II will give an overview of both diagnostics. Section III addresses the degeneration of the diodes and validates the diode measurements by a comparison to the foil measurements on consecutively shorter time scales. In section IV, the characteristics of ELM induced radiation and the rotation of the post-ELM filaments are briefly discussed, taking advantage of the high time resolution of the newly installed diagnostic.

\section{DIAGNOSTICS}

Bolometric diagnostics measure the radiated energy emitted along a line of sight. They are typically sensitive to a wide range of photon energies, from visible light $(\sim 1 \mathrm{eV})$ to soft $\mathrm{X}$-ray radiation (several keV). For an absolutely calibrated measurement of the radiation energy, it is necessary to have a constant energy response. In 
fusion devices, such as AUG, the detectors are installed in the vacuum vessel using pinhole cameras, since any optics would absorb parts of the spectrum.

\section{A. AXUV diode diagnostic}

The new AXUV diode system aims to measure the total radiation using AXUV16ELG semiconductors from International Radiation Detectors, Inc. (IRD) ${ }^{10}$. Photons penetrate the diodes and create electron-hole pairs in the active region (n-on-p junction). The resulting photocurrent should, in principle, be proportional to the incident radiation power ${ }^{11,12}$. AXUV diodes offer a time resolution of less than $500 \mathrm{~ns}^{13}$.

The IRD diodes are specially optimized to measure VUV photons, since standard diodes are not sensitive to this wavelength region. The VUV sensitivity is mainly achieved via surface effects in the top layer of the diode. But, the semiconductor characteristics of the diodes still result in a lower responsivity for VUV light than for other photon energies. Above $\sim 200 \mathrm{eV}$ the responsivity is roughly constant and the diodes are therefore appropriate for bolometric measurements in this photon energy range. The spectral responsivity can be seen in figure 1 .

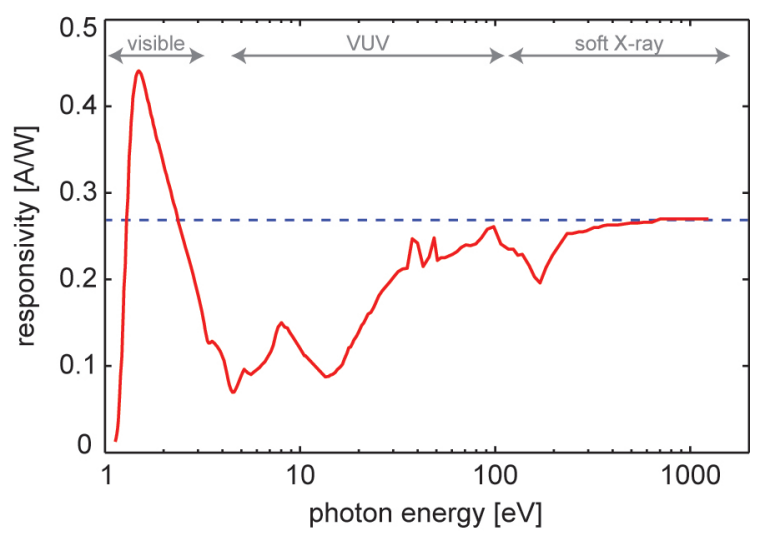

Figure 1. Photon energy dependence of the responsivity of the AXUV diodes (manufacturer specification ${ }^{10}$ ), the calibration value of $0.27 \mathrm{~A} / \mathrm{W}$ is indicated by the dashed line.

Unlike foil bolometers, AXUV diodes are neither sensitive to neutral particles with energies below $200 \mathrm{eV}$ nor pressure changes. It is possible for electrons, ions, neutrons and higher energetic neutral particles to create electron-hole pairs in the diodes and thereby cause a parasitic signal, but at the positions where the diodes are installed, the electron, ion and higher energy neutral particle fluxes are negligible and the interaction of neutrons with the diodes is weak.

The AXUV diode system at AUG consists of $256 \mathrm{LOS}$ (16 AXUV16ELG arrays) divided into 8 cameras. Most of these cameras have a rectangular pinhole of $0.8 \times 2 \mathrm{~mm}^{2}$ and a distance between the detector and the pinhole between $20-80 \mathrm{~mm}$. Both diagnostics, foil bolometry and
AXUV diodes, cover the complete poloidal cross-section of the torus in order to reconstruct two dimensional radiation profiles (the matter of tomographic reconstructions is to be discussed in detail in a separate publication). Most of the LOS of the foil bolometry were duplicated by the AXUV diodes to enable a detailed comparison between the two diagnostics. The diode diagnostic has one additional vertical camera on the opposite side of the torus for toroidal asymmetry studies and two toroidally viewing arrays in collimator cameras resolving the edge radiation. The layout of the LOS can be seen in figure $2(\mathrm{a}, \mathrm{c}, \mathrm{d})$.

The photocurrent created by the diode is fed through a shielded double-core cable of maximum $15 \mathrm{~m}$ length to the measuring board. Each diode requires its own board, which consists of a transimpedance amplifier, two operational amplifiers, a 4th order low pass Bessel filter $(200 \mathrm{kHz})$ and a 14-bit analog-to-digital converter (ADC). The measuring board applies a bias voltage of $8 \mathrm{~V}$ to the diode to widen the depletion volume, avoid recombination inside the diode and to reduce the response time. An automatic offset correction is adjusted before every discharge to compensate the dark current of the diode. The amplification of the measuring board can be adjusted between discharges to obtain a high dynamic range with a maximum current range of $15 \mathrm{~mA}$ and a minimum resolution of $50 \mathrm{nA} .16$ measuring boards are combined in one electronics rack, which is controlled and read out by the SIO data acquisition system ${ }^{14}$ developed at IPP Garching. The SIO system offers a maximum sampling rate of $2 \mathrm{MHz}$. However, the actual sampling rate of the data acquisition is reduced to $500 \mathrm{kHz}$ (in line with the $200 \mathrm{kHz}$ filter on the boards) to allow data from more LOS to be recorded.

\section{B. Foil bolometry}

Foil bolometry is based on radiation absorbing foils. The energy of the incident photons is absorbed by a front layer and converted into heat. Foil bolometers are made out of a thin foil of chemically inert metal (e.g. gold and platinum) placed over an isolating substrate such as Kapton, mica or silicon nitride ${ }^{1-3}$. A meander like structure on its backside acts as a resistor $(\sim 1200 \Omega)$. By measuring its resistance change, the temperature increase and corresponding power deposition on the front of the foil can be calculated ${ }^{15}$.

Since the foils absorb practically all the energy of the photons, the measurement has a linear response to the photon energy and can easily be absolutely calibrated by its temperature characteristics. The sensitive photon energy region is limited on one side by the reflection of low energy photons $(\leq 1 \mathrm{eV})$ on the metal surface and on the other side by the passing of high energetic photons $(\geq 8 \mathrm{keV})$ through the absorber.

The foils used at AUG have an absorptive layer that is $4.5 \mu \mathrm{m}$ thick and a surface area of $5 \mathrm{~mm}^{2}$. The substrate 

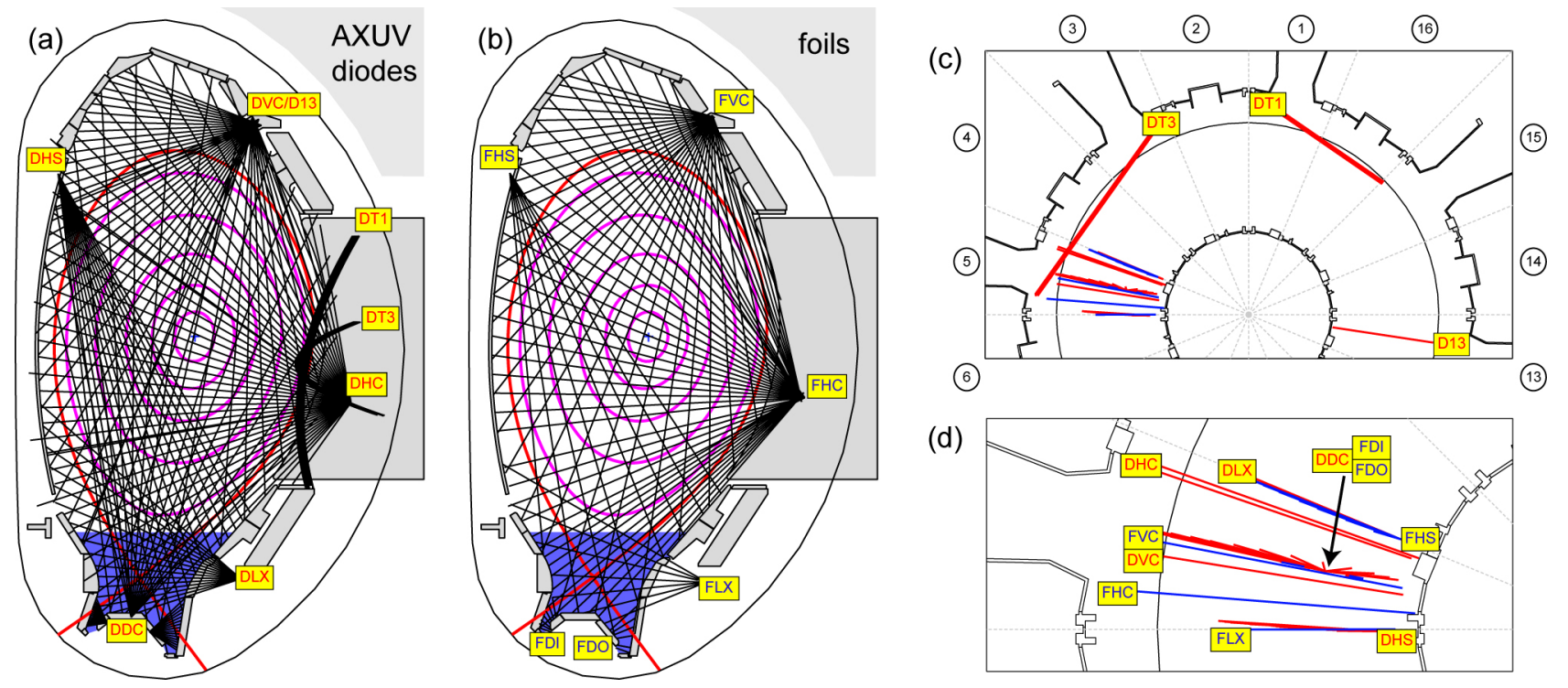

Figure 2. Poloidal cross-section of AUG including the LOS of the diode diagnostic (a) and foil bolometry (b). The divertor region, where most of the power is radiated, is shaded in blue. The red line indicates the last closed flux surface (LCFS) and the magenta lines the flux surfaces in the confined plasma. (c) Toroidal cross-section of AUG (sectors 13-6) including the LOS of the diode diagnostic (Dxx, red) and the foil bolometry (Fxx, blue). (d) Zoom into toroidal cross-section (sector 5).

is between $1.5-20 \mu \mathrm{m}$ thick $^{1-3}$.

The maximum achievable time resolution of the diagnostic is set by the heat transport time from the absorbing foil to the meander, typically on the order of tens of us. The achieved time resolution with the actual setup at AUG is around $2 \mathrm{~ms}$.

The foil bolometry at AUG consists of 112 lines of sight (LOS) installed in pinhole cameras at different poloidal positions in the vacuum vessel. Figure 2(b-d) illustrates the positions of the LOS.

\section{SENSITIVITY DEGRADATION AND VALIDATION OF THE DIODE MEASUREMENTS}

The diode diagnostic and the foil bolometry both aim to measure the total radiation and provide complementary data. According to figure 1 it is expected that diodes measure a lower signal than foil bolometers due to the lower VUV sensitivity.

The environment of a tokamak experiment contains several hazards for semiconductors such as intense VUV radiation, which depletes the top layer of the diode, neutrons and hard X-rays, which can damage the crystal structure of the semiconductor.

With respect to the use of diodes in VUV (i.e. EUV) lithography, sensitivity degeneration of different types of diodes due to VUV irradiation has been reported ${ }^{16}$. Nevertheless, these measurements do not lead to the same results as obtained in the environment of a tokamak plasma. A detailed analysis of the degradation in a tokamak environment is the subject of the subsequent section.

\section{A. Time scale of the degradation}

For the AUG experimental campaign which started in November 2010, all of the cameras were equipped with new diodes and the evolution of the diode signal compared to the foil bolometry was observed in detail.

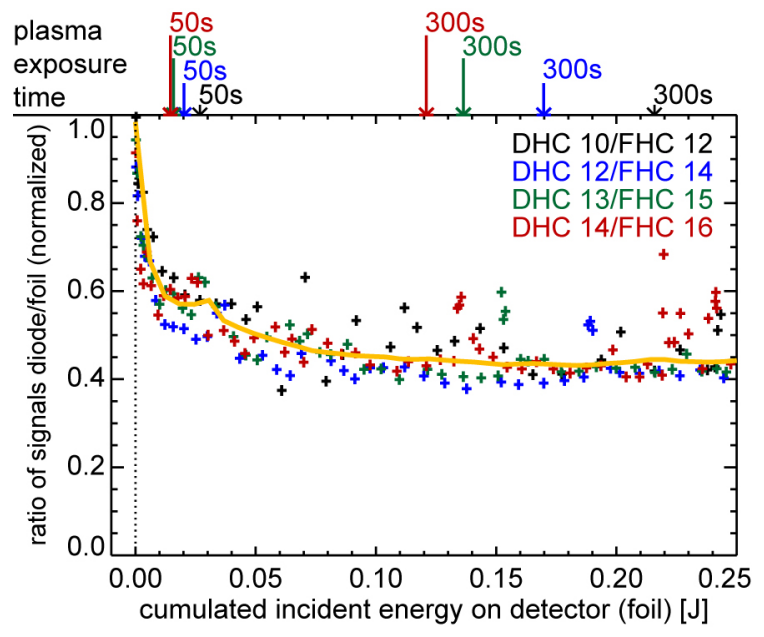

Figure 3. Discharge-averaged normalized ratio of diode measured signal to foil measured signal from 4 LOS doublets, which are mainly observing the confined plasma, plotted over the time history of the cumulated deposited radiation energy. The time the diodes were exposed to the plasma radiation is shown on top.

In figure 3, the ratios of LOS doublets of diode and foil diagnostic are plotted against the total deposited radiation energy. The doublets are located above the divertor 
to have no influence from the divertor radiation. The diode sensitivity decreases strongly within 50 seconds of plasma operation. After roughly 300 seconds of plasma operation the sensitivity remains constant and the observed diode signals are a factor of 2.5 lower than the foil measurements. The diode sensitivity does not change significantly for the next 1000 discharges $(\sim 3000$ s of plasma operation) after this initial degradation.

This indicates a saturating behaviour of the degeneration of AXUV diodes. A similar behaviour of other types of diodes is reported in ${ }^{16}$.

Since the degradation just takes place within the first few plasma discharges, the storage and installation of the diodes and the baking of the vacuum vessel (one week at $150^{\circ} \mathrm{C}$ ) can be excluded as causes of the degradation. The neutron rate is rather low $\left(<10^{12}\right.$ neutrons/s $)$ for the first discharges of the campaign. Therefore, neutrons can also likely be excluded as the cause of the degradation. Highly energetic radiation can be excluded because diodes, which were installed in the divertor and thus not exposed to photon energies above several hundred $\mathrm{eV}$, also degraded. Altogether, this suggests that radiation, especially VUV radiation, is the cause of the degradation.

The behaviour observed in figure 3 suggests that the surface of the diodes depletes rapidly due to the VUV radiation and cannot recover. Therefore, the effect of the VUV absorption, for which these diodes were specially optimized, is canceled. As soon as the surface is fully depleted, the sensitivity no longer changes, resulting in the saturating degeneration as observed.

This fast degeneration is observed for all of the diode channels and is mainly dependent on the total incident radiation. Whether this is an effect of the overall radiation or only the VUV radiation cannot be determined from the available data, since the degradation takes place on a short time scale.

The difference between the signals measured by the diodes and the foils after the initial degradation is changing with the location of the LOS doublet and is higher in the divertor than in the main chamber. The required scaling factor likely depends on the fraction of VUV light of the total radiation along the LOS. Hence the sensitivity must have another spectral dependence due to the degradation rather than being lowered by a constant factor for all photon energies. In the following section (III B) it is shown how the spectral responsivity changed and that it is the same for all diodes, as expected from a saturating degradation.

After longterm operation, severe damage of individual diodes may become apparent. The capacitance of the diodes might change or the dark current may begin to vary on the timescale of individual discharges. These effects can only be detected by comparison of two overlapping LOS of different diode arrays. At AUG, 4 out of 16 diode arrays (or individual diodes from these arrays) encountered these types of damage after 2.5 years of operation. Therefore, it is recommended to replace the diodes at least every second year.

\section{B. Degraded responsivity}

In the above section it was shown that diodes undergo a strong degradation. Since the sensitivity is constant after the initial degradation, one can recalibrate the diode signals with degraded responsivity in order to retrieve useful data.

Several diodes, which were installed for the duration of one campaign (>1600 discharges, 4800 seconds plasma operation) at different positions in the vacuum vessel, have been post calibrated by the PhysikalischTechnische Bundesanstalt (PTB) in Berlin. The spectral responsivity of these diodes was measured at the synchrotron BESSY for photon energies between $50 \mathrm{eV}$ and $1600 \mathrm{eV}^{17,18}$. A calibration for VUV light with photon energies below $50 \mathrm{eV}$ was not possible since the generated photocurrent was on the order of the dark current fluctuations (due to the low brilliance of BESSY in the VUV range).

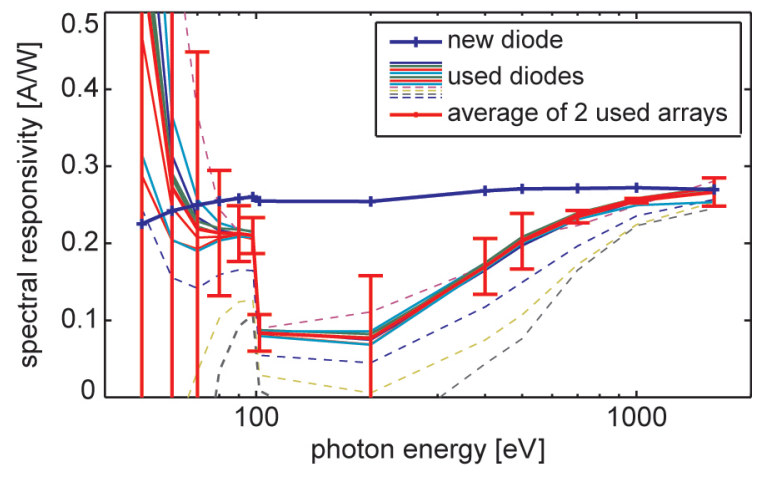

Figure 4. Degraded responsivity of several diodes out of different arrays, averaged responsivity (red) and initial responsivity (blue), measured by the PTB.

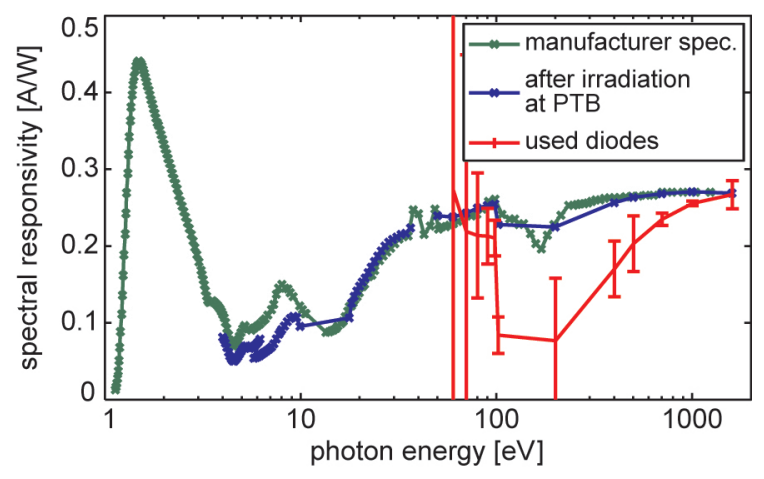

Figure 5. Averaged degraded responsivity (red), initial responsivity $^{10}$ (green) and responsivity after irradiation with 70-100 eV photons, measured by the PTB.

In figure 4 the measured responsivity of a new diode (blue line) is compared to the measurement of selected degraded diodes. Most of the diodes show the same degraded responsivity, even though they were installed at 
diverse positions in the torus and observed different regions of the plasma. The degraded responsivity is independent of the exposed photon energies and neutron and photon fluxes. This is explained by the saturating degradation.

The diodes from one array in particular (dashed lines in Fig. 4) exhibit increased fluctuations of the dark current, inhibiting detailed measurements of the responsivity. These fluctuations may be caused by damages due to the long term operation of the diodes, mentioned in the previous section.

While the sensitivity to high energy photons $(>500 \mathrm{eV})$ and photons with energies between $70-100 \mathrm{eV}$ is only weakly decreased, there is a strong degradation between $100 \mathrm{eV}$ and $\sim 400 \mathrm{eV}$. The increase of responsivity below $70 \mathrm{eV}$ is not physical and is created by the large error bars of the measurements at low photon energies.

The measurements at the PTB included an irradiation of new diodes with photons of $70-100 \mathrm{eV}$ to estimate the contribution of this energy range to the degradation. This irradiation had an overall power deposition of $0.9 \frac{\mathrm{J}}{\mathrm{mm}^{2}}$, much higher than the total power deposition during one campaign at AUG. Figure 5 compares the responsivity of a new (green), irradiated (blue) and previously installed diode (red). The responsivity of the controlled irradiated diode did not change significantly above $10 \mathrm{eV}$ and decreased slightly between $4-10 \mathrm{eV}$. In comparison to the measurements of the installed diodes, the observed damage (between $100 \mathrm{eV}$ and $\sim 400 \mathrm{eV}$ ) cannot be caused by the photon energies of the irradiation.

Unfortunately, the sensitivity in the VUV range could not be measured. However, there are several indications that the sensitivity in the VUV range is strongly reduced. The controlled irradiated diodes (irradiated with photon energies of $70-100 \mathrm{eV}$ ) show a reduction between $4-10 \mathrm{eV}$ and no effect for higher photon energies. This indicates that the sensitivity for the VUV region (here $4-10 \mathrm{eV}$ ) is susceptible to damage, also from photons of other energies. The previously installed diodes are more strongly degraded between $100 \mathrm{eV}$ and $\sim 400 \mathrm{eV}$ than the controlled irradiated ones. We assume that these diodes are also more severely affected in the VUV range. This is consistent with the fact, that the deviation between foil and diode measurements is much stronger in the divertor region (see Sec. IIIC), where a higher fraction of VUV light is emitted. The deviation for LOS in the divertor cannot be explained by a reduction of the sensitivity only above $100 \mathrm{eV}$.

There are also no calibrated measurements of the sensitivity to visible light $(\sim 1-3 \mathrm{eV})$, but the degraded diodes still generate a significant photocurrent when irradiated by a visible light source. However, this photon energy range has only a very small impact on the bolometric measurements since most of the power is irradiated at higher photon energies.

Overall, all diodes seem to have the same sensitivity after the initial degradation, independent of their mounting position or observed plasma region. We as- sume a strong decrease of sensitivity for VUV radiation (between 3-50 eV). A severe degradation for photons between $\sim 100-400 \mathrm{eV}$ was measured. The sensitivity for visible light $(\sim 1-3 \mathrm{eV})$, between $50-100 \mathrm{eV}$, and above $400 \mathrm{eV}$ is only marginally affected. The diodes remain sensitive for these three radiation bands. The observed degradation is not caused by photons between $70-100 \mathrm{eV}$ or above several hundred $\mathrm{eV}$ or neutrons. However, it cannot be determined if the damage of the diodes is from radiation in the VUV range or from photon energies of $100-500 \mathrm{eV}$.

\section{Comparison to foil measurements}

The measurements of the AXUV diodes need to be validated against the foil bolometry. Therefore, the nonlinear wavelength dependence has to be taken into account, as it is presented for specific applications in references ${ }^{5,6}$. The references point out the strong impact of radiation in the VUV region, as anticipated from the initial diode responsivity. The divertor radiation, which is dominated by VUV light and generally outshines the main chamber radiation in a tokamak, is omitted in these analyses. Moreover, the degraded sensitivity, as described in the previous section, introduces an additional discrepancy for higher energetic photons. Here, an attempt to compare diode and foil measurements including the divertor region and ELM induced radiation is presented.

In figure 6, the measurements of LOS doublets of diode and foil diagnostics at two positions are compared throughout one discharge. The positions are chosen to compare LOS through the core with low VUV contribution and more high energy photons (left side) and through the divertor with dominating low energy radiation below $100 \mathrm{eV}$ (right side). The core LOS needs a lower scaling factor for the degradation (2.5) than the divertor LOS (10).

The top plots show the evolution during the whole discharge. The corrected diode signals measure similar signals as the foil bolometry. This suggests that a constant scaling factor over the whole discharge can be applied. The discrepancies $(\leq 30 \%)$ are caused by a weakly temporally evolving VUV background. The scaling factor might still differ between discharges.

The middle row of figure 6 shows a short time period of the same measurements highlighting the full time resolution of the diagnostics. The main discrepancies between the signals occur during the radiation peaks, which are caused by ELMs. By reducing the time resolution of the diodes (averaging over $3 \mathrm{~ms}$, bottom of Fig. 6), both measurements agree within their uncertainties. They measure the same radiated energy of an ELM along the LOS, even though the radiation of the plasma is changing significantly due to ELMs (a high fraction of the ELM energy is emitted by radiation in the divertor).

The changing radiation due to an ELM does not influence the cross-calibration of the diodes with the foils. This suggests that the diodes can also be used to an- 

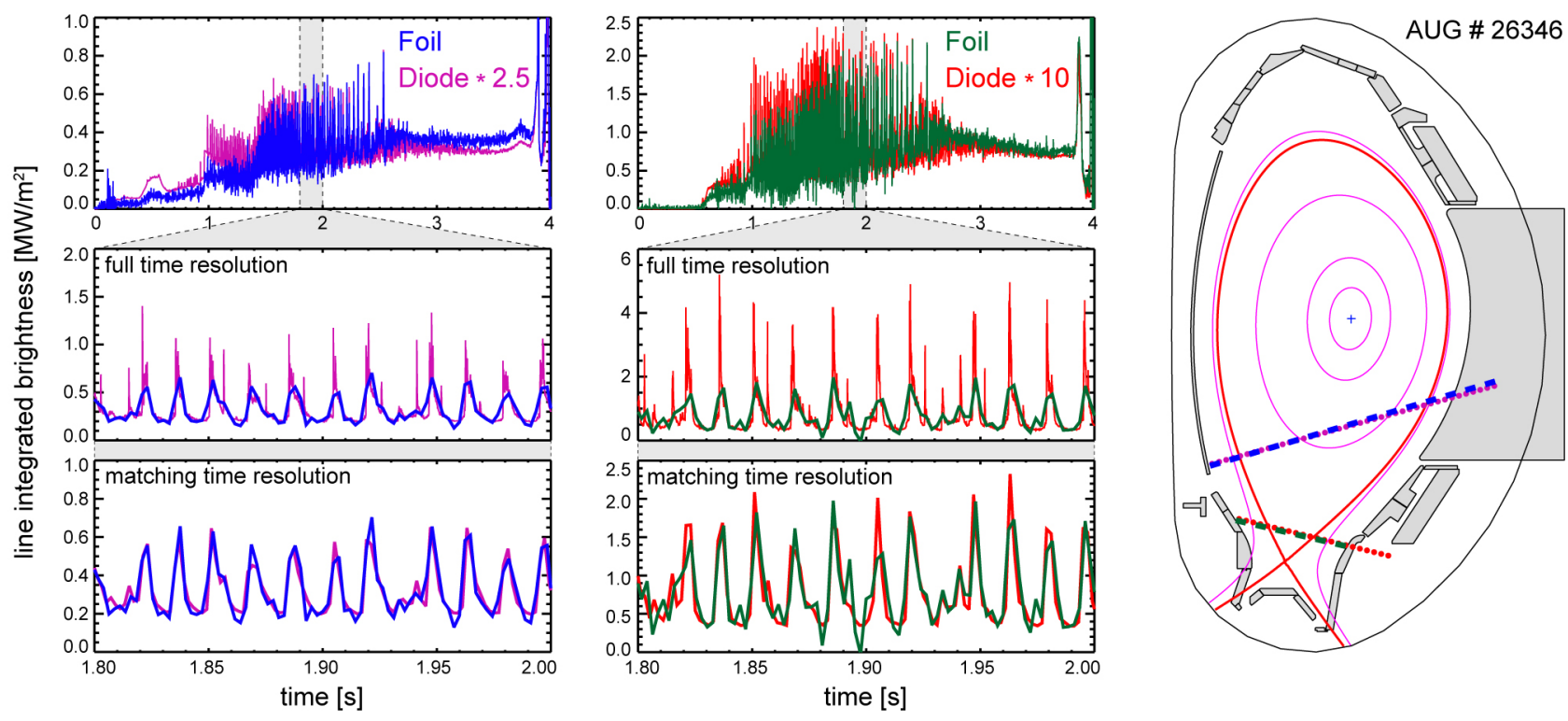

Figure 6. Comparison of time traces of LOS doublets of diode and foil diagnostics (AUG discharge 26346). The diode measurements are scaled with a fixed factor to compensate for the effects of degradation. The middle plots show ELM signals measured by both diagnostics in full time resolution. For the bottom plots the diode and foil signals are averaged over 3 ms to match their time resolution.

alyze absolutely calibrated ELM radiation with a time resolution of down to 5 ss.

\section{APPLICATION OF AXUV DIODE DIAGNOSTIC}

The AXUV diode diagnostic offers a high time resolution combined with full coverage of the poloidal crosssection of AUG. Therefore, this diagnostic is well suited to study fast radiation events. AXUV diodes themselves are not suited to measure the total radiated power. This can only be determined by the foil bolometry on longer timescales. The combination of both diagnostic provides absolute measurements, extrapolated to shorter timescales.

In reference ${ }^{8}$ the analysis of radiation emitted in mitigated disruptions is discussed. The temporal evolution and poloidal asymmetries of the ELM induced radiation can also be studied in detail, using the AXUV diodes. As an example, the substructures of ELM induced radiation in the divertor region are analysed in the following section, taking advantage of the unique capabilities of the newly installed diagnostic.

\section{Rotation of post-ELM filaments}

ELM correlated, spatially localized radiation peaks are observed by the diode measurements in the inner divertor. Their dynamics are well resolved by the diagnostic as shown in figure 7. Similar observations are reported in reference ${ }^{19}$. The initial ELM induced radiation is almost completely decayed after $0.5 \mathrm{~ms}$, however filamen- tary structures are observed for almost $2 \mathrm{~ms}$ at the inner divertor. The LOS of the AXUV system, which observe these post-ELM filaments, are shown in figure 8 .

ELMs are reported to produce field-aligned structures of high plasma pressure, called filaments, in the $\mathrm{SOL}^{20}$, which originate from the pedestal. These filaments are radially, poloidally and toroidally localized but have a life time of only a few hundreds of $\mu$ s and toroidal mode numbers in the range of 4 to $14^{21,22}$.

The post-ELM filaments observed by the AXUV diagnostic might be remanent structures of the ELM filaments ${ }^{20-22}$ or might be correlated to the X-point fluctuations reported in $^{23}$, for which the radiation might be intensified by the ELMs.

ELM filaments rotate toroidally ${ }^{22}$. Similar observations can be made with the post-ELM filaments. Given the toroidal location of the measurements of the AXUV diodes, they appear at different times depending on their toroidal phase and rotation velocity. Long lasting filaments might reappear at the same location after one rotation period ${ }^{24}$.

The setup of the diode diagnostic includes two cameras at toroidally opposing positions with the same poloidal viewing geometry (DVC and D13, in Fig. 2). They provide the opportunity to compare signals from both sectors and check for toroidal asymmetries and toroidal transport time scales.

The time of the appearance of a filament in the opposite sectors is illustrated in figure 9 . The signal from sector 5 (DVC) is shown in red and the signal from sector 13 (D13) in blue. Both LOS observe the inner divertor. The filament peaks in the two sectors are well correlated.

The time differences between the appearance of the fil- 


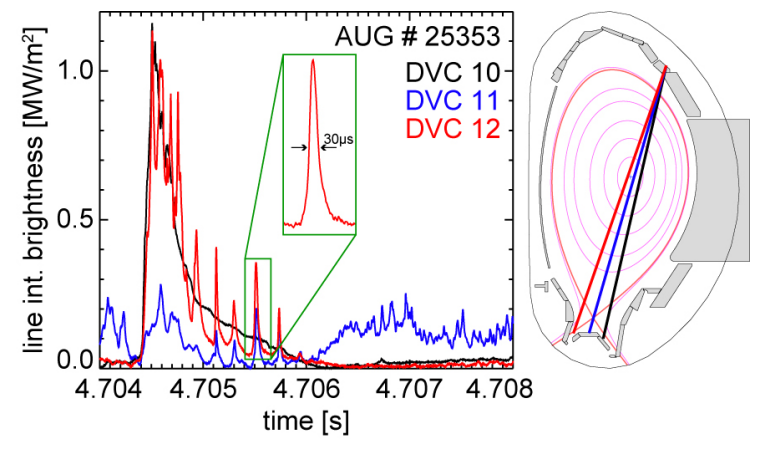

Figure 7. Measured ELM radiation from three LOS of the vertical camera observing the outer divertor (black), X-point region (blue) and inner divertor (red) (AUG discharge 25353). While the absolute radiation is comparable, the inner divertor shows a detailed structure of so-called post-ELM filaments, which in this case appear after the initial radiation peak and might be remanent structures of the ELM. The duration of these features of around $30 \mu \mathrm{s}$ is well within the time resolution of the diagnostic.

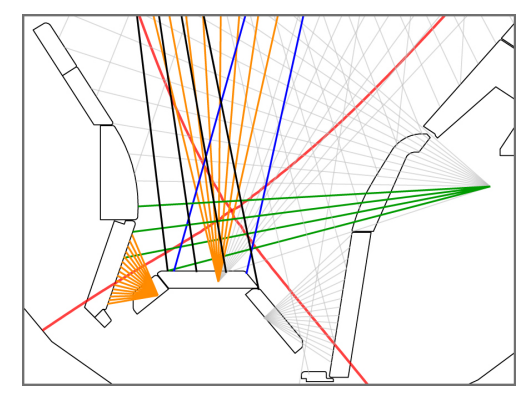

Figure 8. Cutout of the divertor including the positions of the LOS of several AXUV cameras, which observe the post-ELM filaments in AUG discharge 29874. The LOS, which do not observe the filaments are indicated in gray, the separatrix in red.

ament in sector 13 and its reappearance in sector 5 are listed in figure 9 , as well as the time differences between the appearance in sector 5 and the reappearance in sector 13. In the beginning both time delays are around $\Delta t=80-90 \mu \mathrm{s}$. Over the duration of roughly $0.5 \mathrm{~ms}$ they approach a constant value of about $\Delta t=130 \mu \mathrm{s}$. Since the two sectors are 180 degrees apart, the matching time differences indicate a toroidal rotation velocity of a structure with an odd toroidal mode number $(n=1,3,5, \ldots)$. Assuming the measured peaks are caused by a single quasi-stable filament $(n=1)$, its average rotation frequency is estimated:

$$
f_{\text {rot }, \text { tor }}^{\text {filament }}=\frac{n}{T}=\frac{n}{2 \cdot \Delta t}=\frac{1}{2 \cdot 130 \mu \mathrm{s}} \approx 3.8 \mathrm{kHz} .
$$

The impurity ion toroidal rotation velocity obtained by the charge exchange recombination spectroscopy diagnostic (CXRS) is $50 \pm 15 \mathrm{~km} / \mathrm{s}$ at the top of the pedestal on the low field side midplane. This corresponds to a

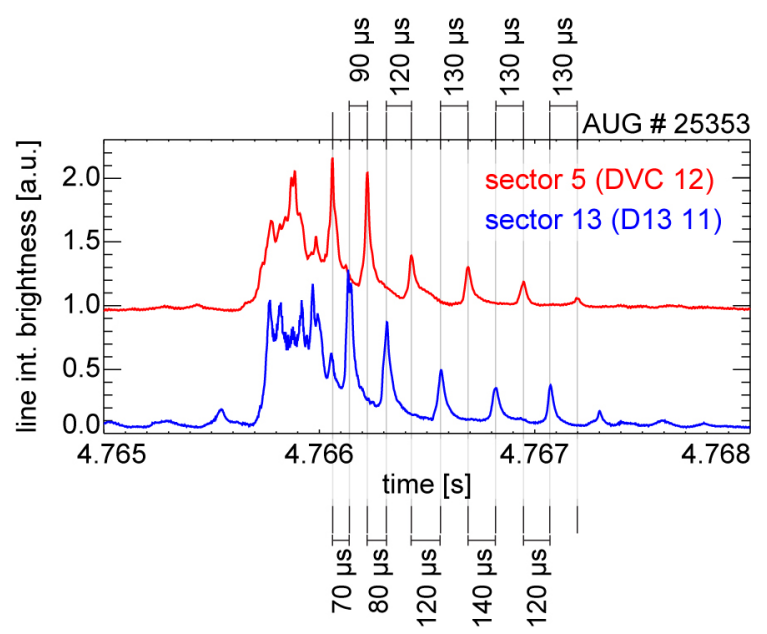

Figure 9. ELM induced radiation at the inner divertor in two opposite sectors of AUG (sector 5 (red) and sector 13 (blue)). The toroidal rotation of a post-ELM filament is observed. The time delay of the appearance of the filament in sector 13 and the reappearance in sector 5 are noted on top, the time delay between sector 5 and sector 13 on the bottom. For better visibility, an offset is added to the red curve.

rotation frequency of

$$
f_{\text {rot,tor }}^{\mathrm{CXRS}}=\frac{v_{\text {rot,tor }}}{2 \pi R}=\frac{50 \pm 15 \mathrm{~km} / \mathrm{s}}{2 \pi 2.1 \mathrm{~m}} \approx 3.8 \pm 1.1 \mathrm{kHz},
$$

which is in good agreement with the calculated rotation frequency of the filament.

If the filament were completely detached from the confined plasma, its energy would be transported to the plasma facing components within less than $100 \mu \mathrm{s}$. The parallel energy transport during ELMs on open field lines is dominated by the ion sound speed at the pedestal top $\left(c_{s, \text { ion }}=\sqrt{\frac{\left(T_{i, p e d}+T_{e, p e d}\right)}{m_{i}}} \approx \sqrt{\frac{2 \cdot 700 \mathrm{eV}}{2 u}} \approx 260 \mathrm{~km} / \mathrm{s}\right)$ and the connection length between the midplane and the divertor target plates $\left(L_{c} \approx 20 \mathrm{~m}\right)^{20,25}$ :

$$
\tau_{\|, E L M} \approx \frac{L_{c}}{c_{s, i o n}} \approx \frac{20 \mathrm{~m}}{260 \mathrm{~km} / \mathrm{s}} \approx 7.7 \mu \mathrm{s}
$$

However, the filament exists more than $1 \mathrm{~ms}$. This duration could result from lower ion temperatures and negligible electron temperatures in the filament $\left(T_{i}=\right.$ $30 \mathrm{eV} \hat{=} \tau_{\|, E L M} \approx 500 \mu \mathrm{s}$ ), but the observed energy losses by radiation would cool down the filament very fast. Therefore, there must be an ongoing energy flux to the filament.

We assume this energy loss channel is created by a change of the magnetic edge topology due to the ELM $^{26-29}$, connecting the edge plasma with the divertor. A similar topology is observed when external or internal magnetic perturbations are present, e.g. with MP coils or locked modes ${ }^{30}$. The change of the magnetic field might be created by edge current structures, which rotate with 
the pedestal top. In this way, the location of the loss channel rotates toroidally with the plasma pedestal top velocity. The toroidal solid body rotation of the plasma is hence causing the observed filamentary radiation characteristics in the divertor. The current diffusion time scale of around $2.5 \mathrm{~ms}$ is in the order of the life-time of the filament.
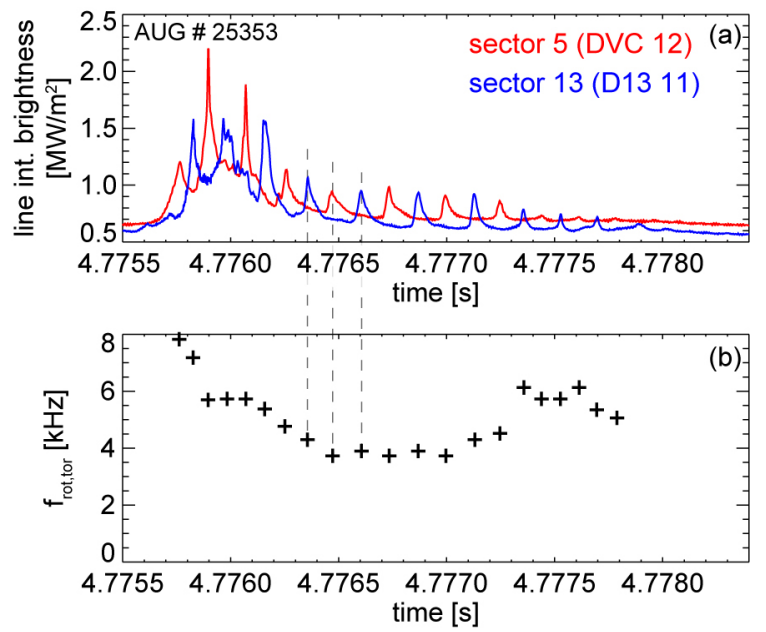

Figure 10. ELM induced radiation at the inner divertor ((a), sector 5 (red) and sector 13 (blue)), and estimated toroidal rotation frequency of the filament (b)

CXRS measurements of $C^{6+}$ show that the toroidal rotation slows down due to an $\mathrm{ELM}^{31}$, in agreement with our presented observations. The same observations are shown in figure 10 for another ELM. Here, an increase of the rotation frequency also can be seen at the end of the ELM cycle, as the gradients of the pedestal build up again. This is also in-line with the CXRS measurements in reference ${ }^{31}$.

This filament rotation analysis has only been performed for a few ELM cycles and, therefore, does not necessarily represent general filament behavior. However, these post-ELM filament peaks are observed quite regularly and a statistical database of filament rotation could be assembled, as this diagnostic is routinely operated at AUG.

\section{SUMMARY}

An extensive set of AXUV diodes was recently installed in ASDEX Upgrade. The AXUV diodes offer radiation measurements of a wide spectral range with a very high time resolution of down to $5 \mu \mathrm{s}$. A high spatial coverage of a fusion experiment can be achieved with them.

The LOS of the foil bolometry at ASDEX Upgrade were duplicated by the diode system to enable the comparison of the two diagnostics. Diode diagnostic and foil bolometry together are a powerful set of complementary diagnostics. The foil bolometers measures the absolutely calibrated radiation power and can be used to recalibrate the fast diode measurements.

The AXUV diodes, despite being optimized to have linear photon energy response, initially have a lower sensitivity for VUV light and additionally undergo a strong, spectrally dependent degradation of the sensitivity in the environment of a tokamak. The resulting responsivity does not allow exact bolometric measurements due to a lack of sensitivity in the VUV region and for photon energies between $100 \mathrm{eV}$ and $400 \mathrm{eV}$. The degradation takes place within the first 50 seconds of plasma operation and afterwards the sensitivity remains constant. Therefore, it is possible to cross calibrate the diode measurements with the foil bolometry on a shot to shot basis. In this way the diode measurements can provide reliably calibrated data on fast timescales.

This new diagnostic provides the opportunity to analyze fast radiation effects, such as ELM induced radiation, which cannot be resolved with the foil bolometry. For example, the toroidal rotation speed of an ELM filament was measured and shown to be connected with the rotation velocity at the pedestal top.

\section{REFERENCES}

${ }^{1}$ K. F. Mast, J. C. Vallet, C. Andelfinger, P. Betzler, H. Kraus, and G. Schramm, Review of Scientific Instruments 62, 744 (1991).

${ }^{2}$ H. Meister, T. Eich, N. Endstrasser, L. Giannone, M. Kannamüller, A. Kling, J. Koll, T. Trautmann, A. Team, P. Detemple, and S. Schmitt, Review of Scientific Instruments 81, 10E132 (2010).

${ }^{3}$ L. Giannone, D. Queen, F. Hellman, and J. C. Fuchs, Plasma Physics and Controlled Fusion 47, 2123 (2005).

${ }^{4}$ R. L. Boivin, J. A. Goetz, E. S. Marmar, J. E. Rice, and J. L. Terry, Review of Scientific Instruments 70, 260 (1999).

${ }^{5}$ M. L. Reinke and I. H. Hutchinson, Review of Scientific Instruments 79, 10F306 (2008).

${ }^{6}$ D. S. Gray, S. C. Luckhardt, L. Chousal, G. Gunner, A. G. Kellman, and D. G. Whyte, Review of Scientific Instruments 75, 376 (2004).

${ }^{7}$ G. Veres, R. Pitts, M. Wischmeier, B. Gulejova, J. Horacek, and S. Kálvin, Journal of Nuclear Materials 363-365, 1104 (2007).

${ }^{8}$ B. Reiter, G. Pautasso, T. Eich, J. C. Fuchs, L. Giannone, R. D. J. Neuhauser, M. Maraschek, V. Igochine, A. Herrmann, T. Lunt, and the ASDEX Upgrade Team, Europhysics Conference Abstracts (Proc. of the 36th EPS Conference on Controlled Fusion and Plasma Physics, Sofia, 2009) 33E, P1.161 (2009).

${ }^{9}$ H. Zohm, Plasma Physics and Controlled Fusion 38, 105 (1996).

${ }^{10}$ International Radiation Detectors, Inc., 2527 West 237th Street Unit A, Torrance, CA 90505-5243, [online] http://www.irdinc.com.

${ }^{11}$ R. Korde, J. Cable, and L. Canfield, Nuclear Science, IEEE Transactions on 40, 1655 (1993).

${ }^{12}$ E. M. Gullikson, R. Korde, L. R. Canfield, and R. E. Vest, Journal of Electron Spectroscopy and Related Phenomena 80, 313 (1996), proceedings of the 11th International Conference on Vacuum Ultraviolet Radiation Physics.

${ }^{13}$ R. Korde, Manual: $A X U V / S X U V / U V G$, International Radiation Detectors, Inc. (2010).

${ }^{14}$ K. Behler, H. Blank, A. Buhler, R. Cole, R. Drube, K. Engelhardt, H. Eixenberger, N. Hicks, A. Lohs, K. Lüddecke, A. Mlynek, U. Mszanowski, R. Merkel, G. Neu, G. Raupp, M. Reich, W. Suttrop, W. Treutterer, and M. Zilker, Fusion Engineer- 
ing and Design 85, 313 (2010), proceedings of the 7th IAEA Technical Meeting on Control, Data Acquisition, and Remote Participation for Fusion Research.

${ }^{15}$ L. Giannone, K. Mast, and M. Schubert, Review of Scientific Instruments 73, 3205 (2002).

${ }^{16}$ P. Kuschnerus, H. Rabus, M. Richter, F. Scholze, L. Werner, and G. Ulm, Metrologia 35, 355 (1998).

${ }^{17}$ A. Gottwald, U. Kroth, M. Krumrey, M. Richter, F. Scholze, and G. Ulm, Metrologia 43, S125 (2006).

${ }^{18}$ F. Scholze, H. Rabus, and G. Ulm, in Proc. SPIE 2808, EUV, $X-R a y$, and Gamma-Ray Instrumentation for Astronomy VII (1996) pp. 534-543.

${ }^{19}$ J. C. Fuchs, M. Bernert, T. Eich, A. Herrmann, P. de Marné, and B. Reiter, Journal of Nuclear Materials 415, S852 (2011), proceedings of the 19th International Conference on Plasma-Surface Interactions in Controlled Fusion.

${ }^{20}$ R. Pitts, P. Andrew, G. Arnoux, T. Eich, W. Fundamenski, A. Huber, C. Silva, D. Tskhakaya, and the JET EFDA Contributors, Nuclear Fusion 47, 1437 (2007).

${ }^{21}$ T. Eich, A. Herrmann, J. Neuhauser, R. Dux, J. C. Fuchs, S. Günter, L. D. Horton, A. Kallenbach, P. T. Lang, C. F. Maggi, M. Maraschek, V. Rohde, W. Schneider, and the ASDEX Upgrade team, Plasma Physics and Controlled Fusion 47, 815 (2005).

${ }^{22}$ A. Kirk, B. Koch, R. Scannell, H. R. Wilson, G. Counsell, J. Dowling, A. Herrmann, R. Martin, and M. Walsh, Phys. Rev. Lett. 96, 185001 (2006).
${ }^{23}$ S. Potzel, M. Wischmeier, M. Bernert, R. Dux, H. Müller, A. Scarabosio, and the ASDEX Upgrade Team, Nuclear Fusion 54, 013001 (2014).

${ }^{24}$ J. Boedo, D. Rudakov, E. Hollmann, R. Moyer, G. McKee, K. Burrell, T. Evans, A. Leonard, W. West, M. Fenstermacher, M. Groth, S. Allen, L. Zeng, G. Wang, and J. Watkins, Journal of Nuclear Materials 337-339, 771 (2005), pSI-16.

${ }^{25}$ A. Herrmann, T. Eich, S. Jachmich, M. Laux, P. Andrew, A. Bergmann, A. Loarte, G. Matthews, J. Neuhauser, the ASDEX Upgrade team, and the Contributors to EFDA-JET work programme, Journal of Nuclear Materials 313-316, 759 (2003), plasma-Surface Interactions in Controlled Fusion Devices 15.

${ }^{26}$ T. Eich, A. Herrmann, J. Neuhauser, and the ASDEX Upgrade Team, Phys. Rev. Lett. 91, 195003 (2003).

${ }^{27}$ H. Koslowski, B. Alper, D. Borba, T. Eich, S. Sharapov, C. Perez, E. Westerhof, and the JET EFDA contributors, Nuclear Fusion 45, 201 (2005).

${ }^{28}$ A. Punjabi, A. Verma, and A. Boozer, Phys. Rev. Lett. 69, 3322 (1992).

${ }^{29}$ A. Wingen, T. E. Evans, C. J. Lasnier, and K. H. Spatschek, Phys. Rev. Lett. 104, 175001 (2010).

${ }^{30}$ H. Müller, T. Lunt, W. Suttrop, T. Eich, R. Fischer, J. C. Fuchs, A. Herrmann, M. Kočan, P. de Marné, E. Wolfrum, and the ASDEX Upgrade team, Journal of Nuclear Materials, (2013).

${ }^{31}$ E. Viezzer, T. Pütterich, G. Conway, R. Dux, T. Happel, J. C. Fuchs, R. McDermott, F. Ryter, B. Sieglin, W. Suttrop, M. Willensdorfer, E. Wolfrum, and the ASDEX Upgrade Team, Nuclear Fusion 53, 053005 (2013). 